\title{
Performance Analysis of Unified Failure Model for Emerging WiMAX Networks
}

\author{
Rajesh Anbazhagan, Nakkeeran Rangaswamy \\ Department of Electronics Engineering, School of Engineering and Technology, Pondicherry University, Puducherry, India. \\ Email: rajesh@pec.edu
}

Received January $6^{\text {th }}, 2012$; revised January $29^{\text {th }}, 2012$; accepted February $22^{\text {nd }}, 2012$

\begin{abstract}
In this paper, we present a failure model for WiMAX network developed by considering the collision due to contention, unavailability of bandwidth and channel error assuming them as independent events. Using this model, the performance of bandwidth request based on contention resolution with exponential increase and exponential decrease (EIED) backoff is investigated and observed low contention efficiency with high access delay. Hence, we modify the EIED mechanism by computing the backoff factor with average contention window and estimating the response time from the transmission failure. Simulations validate the developed model with modified EIED backoff and shows better performance than conventional scheme.
\end{abstract}

Keywords: WiMAX; Medium Access Control; Contention Resolution; Backoff Mechanism

\section{Introduction}

Worldwide interoperability for microwave access (WiMAX) defined by IEEE 802.16 [1] enables the delivery of high data rates that can satisfy user requirements. However, efficient system design in resource allocation for best effort services is necessary to achieve the desired performance. The resource allocations in WiMAX are done frame by frame and depend on the classes of service of the user. The two main operations carried out by the subscriber station (SS) during resource allocation are ranging and bandwidth request.

The types of bandwidth request mechanism specified in 802.16 standards are polling (contention free access) and contention resolution. Although multiple physical layers are specified in WiMAX standard [1], orthogonal frequency division multiplexing (OFDM) air interface with time division duplexing (TDD) based system design is considered with time division multiple access (TDMA) in uplink subframe and time division multiplexing (TDM) in downlink subframe. The ranging and bandwidth request slots used for contention resolution is found in the uplink subframe.

During uplink channel access, the SS executes contention resolution based bandwidth request for best effort services. In contention access, the SS contends for transmission opportunity (TxOP) to execute bandwidth request. If the SS is successful in obtaining the TxOP, then it proceeds in transmitting the bandwidth request. If unsuccessful, it executes contention resolution with backoff mechanism.

The rest of the paper is organized as follows: Related works on bandwidth request mechanism in WiMAX network is presented in Section 2. The developed analytical model is explained in Section 3. Simulation results are shown in Section 4 and concluding remarks is given in Section 5.

\section{Related Works}

In literature, there are excellent discussions on the issues on contention resolution mechanism and its performance for wireless systems. In this section, we review the work related to WiMAX systems. Authors in [2] made detailed discussions on the issues of truncated binary exponential backoff (TBEB) and its performance. Authors in [3] calculated the number of slots with TBEB until the end of current contention period. Authors in [4] suggested exponential increase and exponential decrease (EIED) contention resolution mechanism in WiMAX networks for initial ranging (IR). Authors in [5] proposed bandwidth allocation to support heterogeneous traffic with different quality of service (QoS) requirements in WiMAX networks. Authors in [6] analyzed an adaptive polling scheme for real-time gamming traffic in IEEE 802.16 (WiMAX) networks.

Several researchers [7-10] carried out contention resolution with TBEB. To the best of our knowledge in WiMAX network, no work has been carried out with EIED backoff for bandwidth request. In addition, the literatures 
available on this topic do not explain any mechanisms to force the SS's to implement a backoff factor with adaptive contention window size and response time based on current channel status.

The main contribution made in this paper is the development of unified failure model for WiMAX network. The existing failure models in [2] and [3] account for collision with either unavailability of bandwidth or channel error. Thus, there is no failure model in literature for WiMAX network that completely accounts all possible transmission failures. Hence, we develop a failure model by including these three events that cause the transmission failure. In addition, we propose modified EIED mechanism for bandwidth request to improve the contention efficiency and reduce the access delay for the developed failure model.

\section{Contention Resolution with Unified Failure Model}

\subsection{Proposed Unified Failure Model}

The probability of failure of the WiMAX system can be characterized by three possible events namely: collisions due to contention, unavailability of bandwidth and channel error. Let, $\mathrm{p}_{\mathrm{f}}$ denotes the probability of failure due to above said three possible events and is given by:

$$
\begin{aligned}
p_{f} & =p_{c}+\left(1-p_{e}\right)\left(1-p_{c}\right)(1-q)^{T_{r}}+p_{e} \\
& +p_{c} p_{e}\left(1-p_{e}\right)\left(1-p_{c}\right)(1-q)^{T_{r}} \\
& -p_{c}\left(1-p_{e}\right)\left(1-p_{c}\right)(1-q)^{T_{r}} \\
& -p_{e}\left(1-p_{e}\right)\left(1-p_{c}\right)(1-q)^{T_{r}}-p_{c} p_{e}
\end{aligned}
$$

where $p_{e}$ denotes the probability of error due to channel, $p_{c}$ denotes the probability of collision due to contention, $q$ denotes the probability of base station (BS) to accept a bandwidth request and $T_{r}$ denotes response time or waiting time.

By rearranging (1),

$$
\begin{aligned}
p_{f} & =\left(p_{c}+p_{e}\right)\left[1-\left(1-p_{c}\right)(1-q)^{T_{r}}\left(1-p_{e}\right)\right] \\
& +\left(1+p_{c} p_{e}\right)-p_{c} p_{e}\left[\left(1-p_{c}\right)(1-q)^{T_{r}}\left(1-p_{e}\right)\right]
\end{aligned}
$$

Let $p_{u}=\left(1-p_{c}\right)(1-q)^{T_{r}}\left(1-p_{e}\right)$ and

$$
p_{c e}=p_{c}+p_{e}-p_{c} p_{e}
$$

where $p_{u}$ denotes probability of unavailability of bandwidth and $p_{c e}$ denotes probability due to collision and channel error. Then the $\mathrm{p}_{\mathrm{f}}$ becomes;

$$
p_{f}=p_{u}+p_{c e}\left(1-p_{u}\right)=p_{c e}-p_{u}\left(1+p_{c e}\right)
$$

The failure model in (3) is derived by assuming the three events as independent to each other. The perform- ance of conventional backoff mechanisms with this unified failure model degrade in terms of contention efficiency and access delay. Hence, to improve the performance we aim to compute the backoff factor (such as to reduce collision) and response time (such as to reduce waiting time) by accounting the proposed failure model.

\subsection{Calculation of $\delta_{\mathrm{I}}$ and $\delta_{\mathrm{D}}$ as a Function of $\boldsymbol{W}$}

The main challenge in designing the backoff mechanism is to avoid overlapping of backoff counter among SSs that result in transmission failure due to collision. Since the probability of failure, in this paper, is derived with three possible events, the backoff mechanism in contention resolution has to consider all these events. Hence, we consider EIED backoff mechanism with varying backoff factor (to avoid overlapping of backoff counter) to minimize the effect of transmission failure. The idea behind conventional EIED backoff is that on collision, each SS exponentially increase its contention window with increment backoff factor and on success, the SS decrement its contention window with decrement backoff factor.

Further, the requirement for enhanced backoff mechanism in WiMAX network is due to type of accessing mechanism. Unlike wireless local area network (WLAN), the SS in WiMAX does not use carrier sense multiple access over collision avoidance (CSMA/CA) before transmitting a request. In WLAN network, the backoff counter is decremented as long as the medium is sensed idle and frozen when the medium is sensed busy. In WiMAX network, the backoff mechanism is carried without carrier sensing. Hence, the backoff counter is decremented without accounting the status of the channel or number of stations in the network. This increases the chance of overlapping of backoff counter among the stations that leads to high probability of collision with TBEB and EIED with constant backoff factor.

The increment backoff factor $\left(\delta_{\mathrm{I}}\right)$ and decrement backoff factor $\left(\delta_{\mathrm{D}}\right)$ in conventional EIED depend on the minimum contention window size $\left(\mathrm{CW}_{\min }\right)$, maximum contention window size $\left(\mathrm{CW}_{\max }\right)$, integers $\mathrm{M}$ and $\mathrm{n}$. The $\delta_{\mathrm{I}}, \delta_{\mathrm{D}}, \mathrm{CW}_{\text {min }}$ and $\mathrm{CW}_{\max }$ are related as $\delta_{\mathrm{I}}=\left(\delta_{\mathrm{D}}\right)^{\wedge \mathrm{n}}$ and $\mathrm{CW}_{\text {max }}=\left(\delta_{\mathrm{I}}\right)^{\wedge \mathrm{M}} \mathrm{CW}_{\text {min }}$. In the proposed model, the values of $\delta_{\mathrm{I}}$ and $\delta_{\mathrm{D}}$ are calculated based on average contention window $(\bar{W})$. The resultant backoff factors follow $\delta_{\mathrm{I}}=(\bar{W})^{\wedge(1 / \mathrm{M})}$ and $\delta_{\mathrm{D}}=(\bar{W})^{\wedge(1 /(\mathrm{Mxn}))}$.

The need for average contention window is twofold. Smaller size of contention window results in greater collision among SSs whereas larger window value results in longer waiting time. Secondly, compared to TBEB, in EIED backoff, the SS can control the backoff value either on collision or on success. The need for such complex backoff is to avoid overlapping of backoff counter. 
However, in the case of conventional EIED with constant backoff factor the backoff mechanism converges to TBEB. Hence, an attempt is made to compute the backoff factor based on individual SSs transmission failure.

In this paper, we compute them with average contention window. The value of $\bar{W}$ is computed from the estimation of contention window size with maximum backoff stage "m". Since the contention is performed with EIED, the $\bar{W}$ is derived as follows,

$$
\bar{W}=\sum_{i=0}^{m} W_{i} p\left(W_{i}\right)
$$

The $W_{i}$ with EIED is given by,

$$
W_{i}=W \delta^{i}
$$

where $\delta$ corresponds to initial backoff factor and $W$ is the size of current contention window. Further, the probability of events can be stated as probability of the corresponding values of contention window. Hence, $p\left(W_{i}\right)$ is formulated as follows,

$$
p\left(W_{i}\right)=p p_{f}^{i}
$$

where $p$ is the probability of transmission. Substituting (5) and (6) in (4), the $\bar{W}$ is given by,

$$
\begin{aligned}
& \bar{W}=\sum_{i=o}^{m-1}\left(W \delta^{i}\right)\left(p p_{f}^{i}\right) \\
& \bar{W}=W P \sum_{i=o}^{m-1}\left(\delta^{i}\right)\left(P_{f}^{i}\right)
\end{aligned}
$$

Representing the probability of transmission $(p)$ in terms of probability of failure $\left(p_{f}\right)$ and solving further, the average contention window with EIED is derived as follows,

$$
\bar{W}=W \frac{\left(1-p_{f}\right)}{\left(1-p_{f}^{m}\right)} \frac{\left(1-\delta p_{f}^{m}\right)}{\left(1-\delta p_{f}\right)}
$$

where $\delta$ and $W$ correspond to initial backoff factor and initial contention window size, respectively. By substituting $\bar{W}$ in $\delta_{\mathrm{I}}$ and $\delta_{\mathrm{D}}$, the backoff factors, finally, take the expression,

$$
\begin{aligned}
& \delta_{I}=\left(W \frac{\left(1-p_{f}\right)}{\left(1-p_{f}^{m}\right)} \frac{\left(1-\delta p_{f}^{m}\right)}{\left(1-\delta p_{f}\right)}\right)^{\wedge} \frac{1}{M} \\
& \delta_{D}=\left(W \frac{\left(1-p_{f}\right)}{\left(1-p_{f}^{m}\right)} \frac{\left(1-\delta p_{f}^{m}\right)}{\left(1-\delta p_{f}\right)}\right)^{\wedge \frac{1}{(M \times n)}}
\end{aligned}
$$

The need for such backoff mechanism with dynamic backoff factor in WiMAX network is to introduce fairness among SSs while contending for bandwidth request.

\subsection{Estimation of Response Time}

Another main challenge in contention resolution mecha- nism is that there is no guarantee for the SS to acquire its TxOP with fixed response time $\left(T_{r}\right)$. Hence, it increases the access delay. The response time is the time over which the SS waits for response from base station after executing the bandwidth request. The response time in conventional systems is constant and it depends on the frame duration. This motivated us to estimate the response time through the developed transmission failure model. The geometric probability distribution is considered in the proposed system [11]. Therefore, the average value of contention window is given by the expected value of random variable. Thus, the probability of transmission $(p)$ with average contention window becomes, $p=2 / W$ and is given by,

$$
p=\frac{2\left(1-p_{f}^{m}\right)\left(1-\delta p_{f}\right)}{W\left(1-p_{f}\right)\left(1-\delta p_{f}^{m}\right)}
$$

The response time can be expressed in terms of $p_{f}, p_{c}$, $q$ and number of contending stations $\left(N_{c}\right)$. Since the events causing the transmission failure are assumed as independent, the $T_{r}$ from (2) can be expressed as follows:

$$
T_{r}=\frac{\log \left[\frac{p_{f}+p_{c}\left(p_{e}-1\right)-p_{e}}{\left\{\left(1-p_{c}\right)\left(1-p_{e}\right)\right\}^{2}}\right]}{\log (1-q)}
$$

Expressing the probability of transmission given in (12) in terms of probability of collision, and solving further the response time can be modeled as follows,

$$
T_{r}=\frac{\log \left[\frac{p_{f}+\left(1-\left(1-\frac{2}{\bar{W}}\right)^{N_{c}-1}\right)\left(p_{e}-1\right)-p_{e}}{\left.\left(\left(\frac{2}{\bar{W}}-1\right)^{N_{c}-1}\right)^{2}\left(1-p_{e}\right)^{2}\right]}\right.}{\log (1-q)}
$$

The contention efficiency $\left(p_{s}\right)$ is the probability that a transmission opportunity containing a successful bandwidth request. The $p_{s}$, in the proposed system, is computed by accounting the channel error, unavailability of bandwidth and collision and hence it can be expressed as follows,

$$
p_{s}=N_{c} p(1-p)^{N_{c}-1}\left(1-p_{e}\right)\left(1-(1-q)^{T_{r}}\right)
$$

The access delay $(D)$ is the average number of frames the SS receives the response from BS. It is given by,

$$
D=N_{c} /\left(T x O P \times p_{s}\right)
$$

The contention efficiency reflects how efficient the SSs in acquiring the channel. Increase in contention efficiency increases the throughput of the system and reduces the access delay. Further, the reduced access delay 
reduces the end-to-end delay of the system.

\section{Model Validation and Performance Evaluation}

In this section, the developed failure model and the contention based bandwidth request mechanism with EIED backoff for WiMAX network are validated through Matlab simulations. The following data are considered for this simulation. The duration of frame is $5 \mathrm{~ms}$, the number of contention slots within a physical frame for bandwidth request is 16 , minimum contention window is 8 , initial value of waiting time (response time) set by the BS is 5 and maximum number of backoff stage is 6 . Also, the MAC parameters are configured in accordance with the standard given in [1].

The system performance is evaluated in terms of contention efficiency and access delay by varying the contending stations for different values of $q$, namely 0.25 , and 0.60 . In addition, all simulations are carried out considering error prone channel for the system with $30 \%$ and $50 \%$ error rates. Figure 1 shows the contention efficiency of bandwidth request mechanism with $30 \%$ error rate. The contention efficiency increases with increase in number of stations and attains saturation. The contention efficiency at this saturation region lies between $24.5 \%$ and 28\% for modified EIED backoff, whereas it lies between $22.4 \%$ and $26.6 \%$ for conventional EIED backoff.

A backoff mechanism can be efficient if it performs well with less bandwidth availability. With more bandwidth, the failure due to unavailability of bandwidth and collision due to transmission over the same TxOP is less significant. It is also witnessed from the graph that the proposed EIED backoff (with estimated response time and backoff factor) performs well when $q$ equals to 0.25 . The proposed method imparts an improvement of 9.94\% than conventional EIED.

With increase in the value of $q$, the major contribution to transmission failure is channel error. Thus, an im-

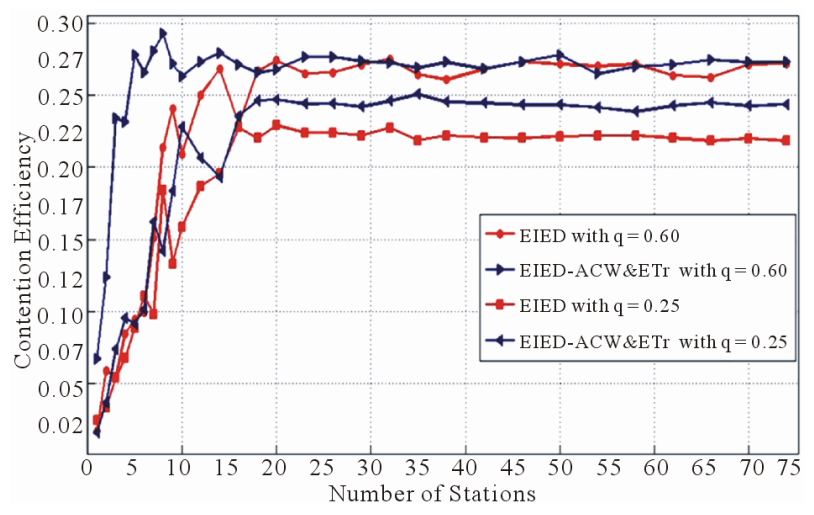

Figure 1. Contention efficiency under varying number of contending stations with $30 \%$ channel error (ACW-average contention window, ETr-estimated response time). provement of $2.39 \%$ is observed when $q$ equals to 0.60 . Simulations carried out with $50 \%$ error rate are also shown in Figure 2, in which, contention efficiency lies between $18.5 \%$ and $22.7 \%$ for modified EIED backoff, whereas it lies between $17.5 \%$ and $21.6 \%$ for conventional EIED backoff. An improvement in contention efficiency of $4.34 \%$ and $6.14 \%$ for the $q$ value of 0.60 and 0.25 respectively, is also noticed.

The access delay of contention based bandwidth request with 30\% error rate is shown in Figure 3. It increases with increase in the number of stations. The conventional backoff experiences high access delay due to longer waiting time (due to fixed response time) and constant backoff factor. The choice of $q$ while computing contention efficiency holds well in the case of access delay. The access delay of the system with conventional EIED is $5.2660 \mathrm{~ms}$ and $6.4551 \mathrm{~ms}$ for the $q$ value of 0.60 and 0.25 respectively, when number of stations equals to 50. The delay is reduced to $5.1344 \mathrm{~ms}$ and $5.8717 \mathrm{~ms}$ with modified EIED. The proposed system shows an

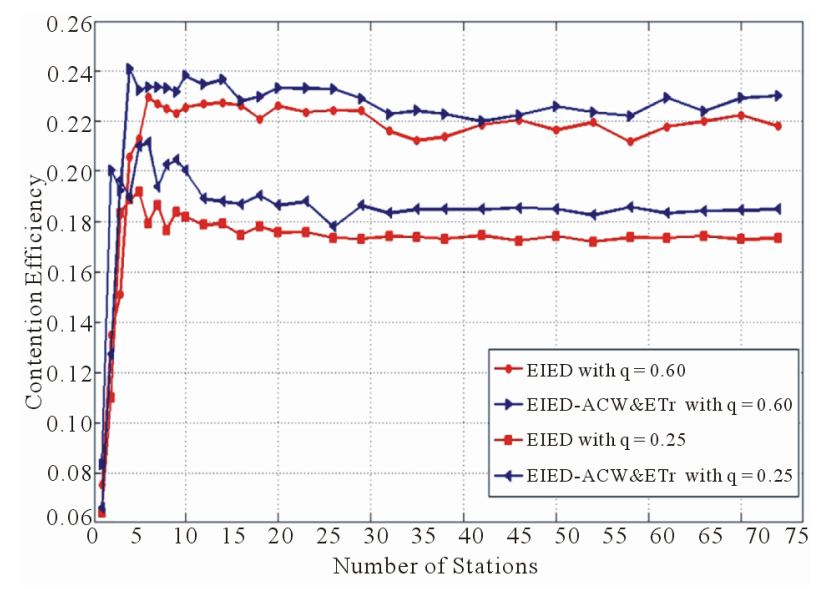

Figure 2. Contention efficiency under varying number of contending stations with $\mathbf{5 0 \%}$ channel error.

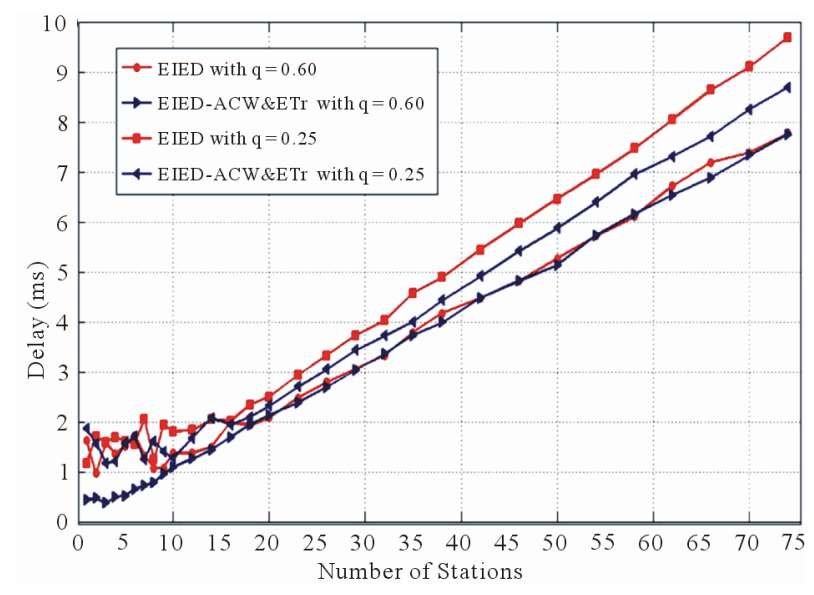

Figure 3. Access delay under varying number of contending stations with $30 \%$ channel error. 


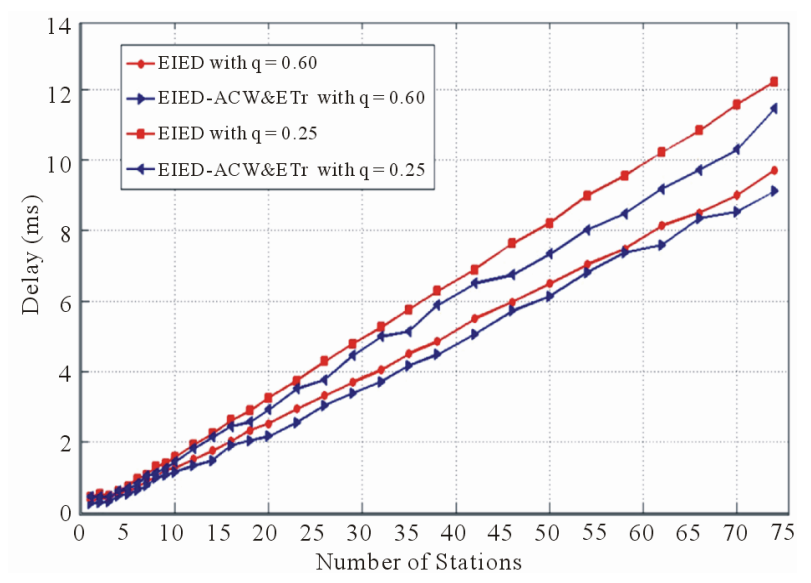

Figure 4. Access delay under varying number of contending stations with $50 \%$ channel error.

improvement of $2.49 \%$ and $6.03 \%$ for the $q$ value of 0.60 and 0.25 respectively than conventional system.

Simulations are also repeated with 50\% error rate and the results are shown in Figure 4. The access delay of the system with basic EIED is $6.4880 \mathrm{~ms}$ and $8.1987 \mathrm{~ms}$ for the $q$ value of 0.60 and 0.25 respectively. The delay is reduced to $6.1356 \mathrm{~ms}$ and $7.3299 \mathrm{~ms}$ in the case of modified EIED. Thus, the proposed system shows an improvement of $5.44 \%$ and $4.59 \%$ with $50 \%$ channel error. We also note that an increase in the value of $q$ with $30 \%$ and $50 \%$ error rate increases the performance of the system nonlinearly that would be due to the independent nature of events that cause transmission failure.

\section{Conclusion}

In this paper, we presented the developed unified failure model for WiMAX network and studied the performance with contention based bandwidth request mechanism for best effort services. Simulations show that the contention resolution with modified EIED backoff performs better than conventional scheme in terms of contention efficiency and access delay under varying error rates. The failure model presented, here, can be extended to design efficient polling mechanisms for real-time services in emerging WiMAX network.

\section{Acknowledgements}

This work is supported in part by DST (Department of Science and Technology), MST (Ministry of Science and Technology), INDIA, under the INSPIRE (Innovation in Science Pursuit for Inspired Research) support program of AORC (Assured Opportunity for Research Career).

\section{REFERENCES}

[1] "IEEE Standard for Local and Metropolitan Area Networks Part 16: Air Interface for Broadband Wireless Access Systems,” 2009, pp. C1-2004.

[2] Q. Ni, L. Hu, A. Vinel, Y. Xiao and M. Hadjinicolaou "Performance Analysis of Contention Based Bandwidth Request Mechanisms in WiMAX Networks," IEEE Systems Journal, Vol. 4, No. 4, 2010, pp. 477-486. doi:10.1109/JSYST.2010.2088770

[3] Y. P. Fallah, F. Agharebparast, M. R. Minhas, H. M. Alnuweiri and V. C. M. Leung, “Analytical Modeling of Contention-Based Bandwidth Request Mechanism in IEEE 802.16 Wireless Networks," IEEE Transactions on Vehicular Technology, Vol. 57, No. 5, 2008, pp. 3094-3107. doi:10.1109/TVT.2007.914474

[4] B.-J. Kwak, N.-O. Song and D. S. Kwon, "Enhancement of IEEE 802.16 WirelessMAN Ranging Performance with EIED Backoff Algorithm,” IEEE 66th Vehicular Technology Conference, Baltimore, 30 September 2007-3 October 2007, pp. 1902-1906.

[5] A. Esmailpour and N. Nasser, "Dynamic QoS-Based Bandwidth Allocation Framework for Broadband Wireless Networks," IEEE Transactions on Vehicular Technology, Vol. 60, No. 6, 2011, pp. 2690-2700. doi:10.1109/TVT.2011.2158674

[6] I. S. Young and D. K. Sung, “Adaptive Real-Time Polling Service for On-Line Gaming Traffic in IEEE 802.16 e/m System,” IEEE Communications Letters, Vol. 14, No. 10, 2010, pp. 987-989. doi:10.1109/LCOMM.2010.081610.100536

[7] Q. Ni and L. Hu, "An Unsaturated Model for Request Mechanisms in WiMAX," IEEE Communications Letters, Vol. 14, No. 1, 2010, pp. 45-47. doi:10.1109/LCOMM.2010.01.091989

[8] Y. Sun, Y. L. Song, J. L. Shi and E. Dutkiewicz, "Research on Bandwidth Reservation in IEEE 802.16 (WiMAX) Networks," Proceedings of IEEE Telecommunications and Malaysia International Conference on Communications, Penang, 14-17 May 2008, pp. 638-643.

[9] J. Delicado, F. M. Delicado, L. Orozco-Barbosa and Q. Ni, "Adaptive Contention Resolution Procedure for Emerging WiMAX Networks," Proceedings of Third Joint IFIP Wireless and Mobile Networking Conference, Budapest, 13-15 October 2010, pp. 1-6.

[10] S.-F. Chou, J.-H. Liu, H.-L. Chao, T.-C. Guo, C.-L. Liu and F.-J. Tsai, "Performance Enhancement of ContentionBased Bandwidth Request Mechanism in IEEE 802.16 WiMAX Networks," Proceedings of IEEE 21st International Symposium on Personal Indoor and Mobile Radio Communications, Istanbul, 26-30 September 2010, pp. 1287-1292.

[11] N. D. Tripathi, J. H. Reed and H. F. VanLandingham, "Radio Resource Management in Cellular Systems," Kluwer Academic Publishers, Plymouth, 2002. 